\title{
Improved fast handover method for multiple node by using mobile nodes guide
}

\begin{abstract}
The fast mobile internet protocol version 6 (FMIPv6) was suggested as a fast handover mechanism over the mobile wireless Internets in order to reduce the handover latency of a mobile node (MN). However, FMIPv6 was originally designed to deal with single MN's. In mobile wireless Internet, a multiple MNs may do a handover at the same time as a consequence of its movement from one network to another new one. This will therefore lead to the bandwidth waste and low handover performance. This paper intends to propose a multiple handover-based mobile node (MHB-MN) control method and an enhanced FMIPv6 mechanism in order to resolve the abovementioned problem. The proposal of such an MHBMN method aims at having one mobile node work as a guide for a group of neighboring MNs. This means that the guide of MN prepares itself for a handover before actually taking the initial steps of the actual handover operation. Based on obtained results, it is plainly observable that by MHB-MN method, the handover initiation time, handover latency and handover control messages can be reduced compared to those of FMIPv6. Furthermore, the contention of the wireless channel for multiple MNs can be improved by the use of fewer control messages. Finally, the paper introduces an analytical model to show that by enabling the MHB-MN method and enhancing the FMIPv6 method, a multiple of nodes can perform rapid handover processes with low handover latency compared to that of the FMIPv6 technique.
\end{abstract}

Keyword: Fast mobile IPv6; Mobile IPv6; Handover operation; Mobility management; Performance analysis 